\title{
Inflammatory Bowel Disease and Takotsubo Syndrome: Cases of Horses or Zebras?
}

\author{
John E. Madias ${ }^{a, b}$ \\ ${ }^{a}$ From the Icahn School of Medicine at Mount Sinai, New York, NY, USA; ${ }^{b}$ Division of Cardiology, Elmhurst Hospital \\ Center, Elmhurst, NY, USA
}

To the Editor,

I read with great interest the report by Mirijello et al. [1], describing the case of an 81-year-old woman with chronic stenosing Crohn's disease (CD) and recurrent takotsubo syndrome (TTS), and providing a review of the literature about inflammatory bowel disease (IBD) and TTS. The exact pathophysiology of TTS is still elusive, but the authors have provided a comprehensive list of the various plausible triggers and pathoetiological mechanisms, which could be instrumental in causing this enigmatic malady. The authors also draw attention to the many common underpinnings of $\mathrm{CD}$ or IBD and TTS [1]. One wonders if it is possible to talk about physical or emotional triggers in such patients with a devastating chronic illness as CD or IBD, or whether it is more suitable to consider a yin and yang conceptualization of a continuous physical/ emotional complementary conglomerate of triggers constantly present, with episodic exacerbations. One also wonders how common is TTS in patients with CD or IBD in general; for example, the presented patient had a most probable occurrence of TTS (i.e., "zebra" episode 3 years earlier (diagnosed erroneously at that time as acute coronary syndrome, i.e, "horse" episode) [1]. In the authors' review of the literature, there is a mention of recurrent TTS in 2 patients with IBD, 1 among 9 patients with CD, and 1 among 4 patients with ulcerative colitis [1]; however, TTS is probably often missed when atypical or mild TTS cases occur [2]. Indeed, a parallel is the frequent (i.e., 28\%) detection of TTS, when prospectively sought after, in patients admitted to an intensive care unit for noncardiac physical illnesses, implementing proactively serial echocardiography [3].

What can be done to enhance our insights about the pathophysiological link between IBD and TTS? First, increase our awareness and index of suspicion that such a link is real and often is manifest by occurrence of definite TTS [1], or "forme fruste" TTS cases [2]. Second, consider employing a currently available technology monitoring the autonomic sympathetic nervous system input to the heart via the stellate ganglia, by recording and analyzing thoracic signals obtained via conventional electrocardiography (ECG) hook-up [4], in patients admitted with exacerbations of IBD. Third, consider having ambulatory patients with chronic IBD, particularly during IBD flare-ups using smartphone-based ECG technology for serial acquisition/transmission of ECGs to be analyzed for hallmarks of TTS (e.g., decrease in the amplitude of QRS voltage, T-wave inversions, or QTc-interval prolongation [5].

\section{Disclosure Statement}

The author has no conflict of interest to declare.

\section{Funding Sources}

No funding was received for this work.

\section{References}

1 Mirijello A, D’Errico MM, Curci S, Bossa F, d'Angelo C, Vendemiale G, et al. Takotsubo syndrome and inflammatory bowel diseases: does a link exist? Dig Dis. 2019 Aug, DOI: 10.1159/000502088.

2 Madias JE. Are there mild forms of Takotsubo syndrome? Int J Cardiol. 2016 May;211:25-6.
3 Park JH, Kang SJ, Song JK, Kim HK, Lim CM, Kang DH, et al. Left ventricular apical ballooning due to severe physical stress in patients admitted to the medical ICU. Chest. $2005 \mathrm{Jul} ; 128(1): 296-302$.
4 Madias JE. A proposal for a noninvasive monitoring of sympathetic nerve activity in patients with takotsubo syndrome. Med Hypotheses. 2017 Nov; 109:97-101.

5 Madias JE. Is there a "chronic Takotsubo syndrome"? Could "smart-phone"-based technology be of aid? Int J Cardiol. 2015;186:297-8.

\section{KARGER}

๑c 2019 S. Karger AG, Basel
John E. Madias, MD, FACC, FAHA

Division of Cardiology

Elmhurst Hospital Center

79-01 Broadway, Elmhurst, NY 11373 (USA)

E-Mail madiasj@ nychhc.org 